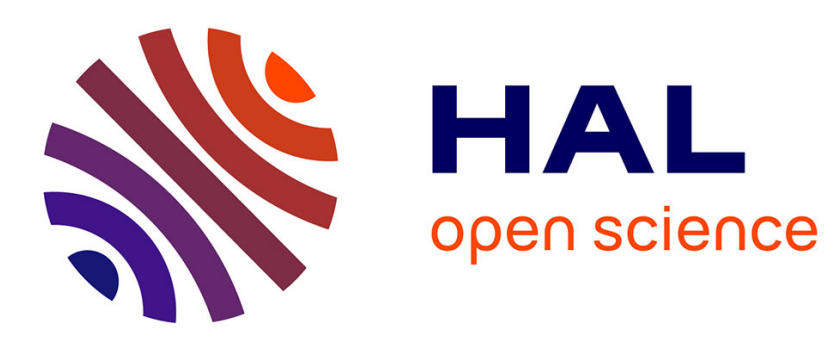

\title{
Alternating Direction Method of Multipliers Framework for Super-Resolution in Ultrasound Imaging
}

\author{
Renaud Morin, Adrian Basarab, Denis Kouamé
}

\section{To cite this version:}

Renaud Morin, Adrian Basarab, Denis Kouamé. Alternating Direction Method of Multipliers Framework for Super-Resolution in Ultrasound Imaging. 9th IEEE International Symposium on Biomedical Imaging: From Nano to Macro (ISBI 2012), May 2012, Barcelona, Spain. pp.1595-1598, 10.1109/ISBI.2012.6235880 . hal-03146719

\section{HAL Id: hal-03146719 \\ https://hal.science/hal-03146719}

Submitted on 23 Feb 2021

HAL is a multi-disciplinary open access archive for the deposit and dissemination of scientific research documents, whether they are published or not. The documents may come from teaching and research institutions in France or abroad, or from public or private research centers.
L'archive ouverte pluridisciplinaire HAL, est destinée au dépôt et à la diffusion de documents scientifiques de niveau recherche, publiés ou non, émanant des établissements d'enseignement et de recherche français ou étrangers, des laboratoires publics ou privés. 


\title{
ALTERNATING DIRECTION METHOD OF MULTIPLIERS FRAMEWORK FOR SUPER-RESOLUTION IN ULTRASOUND IMAGING
}

\author{
Renaud Morin*, Adrian Basarab, Denis Kouamé \\ University of Toulouse \\ IRIT UMR CNRS 5505 \\ Toulouse, France
}

\begin{abstract}
Ultrasound image resolution enhancement is an ongoing challenge to date. Though many works have been performed using device-based approach, there exists few works dealing with post-processing methods. This paper investigates a technique based on the Alternating Direction Method of Multipliers for the resolution enhancement in ultrasound imaging, which includes the deblurring and denoising tasks. We here point out the characteristics of the proposed technique and thereby underline the features that must be dealt with for successful ultrasound image restoration. Synthetic and in vivo ultrasound images are processed in order to asses its performances.
\end{abstract}

Index Terms - Resolution Enhancement, Ultrasound, Medical Imaging, Convex Optimization, Point Spread Function (PSF), Alternating Direction Method of Multipliers (ADMM).

\section{INTRODUCTION}

Ultrasound imaging (USI) is a harmless, cost-effective, noninvasive modality commonly used in the detection of various cancers, in the assessment of blood flow velocity or for investigating biological tissues. Compared with other imaging modalities such as, e.g., X-ray computed tomography, USI only involves an ultrasonic wave and is thus a comparatively safer imaging technique for both the patient and the clinician. It has now become a standard procedure for medical diagnosis such as breast cancer early detection [1, 2]. Unfortunately, due to instrumentation underlying constraints and non-coherent backscattered signals, ultrasound (US) images are contaminated by an intrinsic noise called "speckle" which deeply reduce the general image quality and can lead to inaccurate diagnoses by practitioners.

Compared with other imaging modalities, the main drawback of USI is its poor resolution due to the acoustical characteristics and the geometry of ultrasound transducers. US image resolution improvement is often achieved by optimizing the imaging device, e.g. [3, 4]. An alternative to enhance

\footnotetext{
* Part of this work has been supported by a Région Midi-Pyrénées grant.
}

US image resolution is to investigate post-processing superresolution (SR) techniques. Few works have investigated post-processing techniques and especially the point spread function estimation (PSF) which is still an ongoing challenge today.

Most of recent works are based on deconvolution techniques. Simplest deconvolution methods such as the Wiener and Tikhonov linear filters are quite noise-sensitive and can not take into account the whole characteristics of the PSF. Other works have proposed an extension to the parametric approach [5] and complex deconvolution [6]. Here, we focus on SR in USI and we use the classical image formation model and the inverse problem with a $\mathcal{L}^{2}$ based regularization term, providing smooth solutions. This can be achieved using the $\mathcal{L}^{1}$ total variation (TV) criterion [7] for denoising purposes, thanks to its ability to preserve edges in the image. Recent literature reports efficient methods for general image restoration with the TV criterion based on the Alternating Direction Method of Multipliers (ADMM) [8] approach, which is a variant of the classical Augmented Lagrangian method implementable for optimization problems with separable structures and linear constraints. In this paper, we chose to promote piecewise regular solutions of B-mode ultrasound images within the ADMM framework.

\section{SUPER-RESOLUTION PROBLEM FORMULATION IN ULTRASOUND IMAGING}

\subsection{Image Formation Model}

Under the assumption of weak scattering conditions, the classical interaction between the acoustic field and studied tissues is linear [1] and the ultrasound image formation model is given by the following convolution formulation:

$$
y(t)=g(t) \otimes x(t)+n(t)
$$

where $y(t)$ is the acquired radio frequency (RF) signal, $g(t)$ denotes the PSF, $x(t)$ is the tissue scattering function, $n(t)$ stands for the noise and $\otimes$ represents the convolution operator. It is assumed that the noise has finite energy with a known 
upper bound $\Sigma$.

In this paper, instead of working with real-valued RF signals, we rather work with complex in phase/quadrature (IQ) signals. The IQ signal is an analytic signal obtained via the demodulation of the RF signal in the noise-free case:

$$
\begin{aligned}
z_{I Q}(t) & =y(t)+j \mathcal{H}\{y(t)\} \\
& =g(t) \otimes x(t)+j g(t) \otimes x(t) \otimes \frac{1}{\pi t} \\
& =g(t) \otimes \bar{x}(t),
\end{aligned}
$$

with $j=\sqrt{-1}$ the imaginary unit, $\mathcal{H}\{\cdot\}$ the Hilbert transform and $\bar{x}(t)$ a complex signal to be recovered. Due to the basic principle of USI, the PSF modulated function can be written as $g(t)=h(t) \cos \left(\omega_{0} t+\phi\right)$ with $h(t)$ the envelope of PSF, $\omega_{0}$ a local central frequency and $\phi$ a phase term, the envelope signal $e(t)$ can be written as

$$
e(t)=\left|z_{I Q}(t)\right|=|h(t) \otimes \bar{x}(t)|,
$$

and thanks to the bounded noise assumption, we have

$$
\|e(t)-|h(t) \otimes \bar{x}(t)|\|_{2}^{2} \leq \Sigma .
$$

Here, we introduce in the constrained condition both the phase term in order to remove the absolute operator and the downsampling operator $S$ which accounts for the low resolution nature of $z_{I Q}(t)$ :

$$
\left\|z_{I Q}(t)-S(h(t) \otimes \bar{x}(t))\right\|_{2}^{2} \leq \Sigma .
$$

From (5), we need additional information about $\bar{x}(t)$ to perform its estimation. Some works used the sparsity hypothesis of USI [9] but most of these images are not sparse in the spatial domain. Instead, we investigate here the TV approximation of USI. In this case, the estimation problem within the super-resolution framework becomes

$$
\begin{array}{cl}
\min _{\bar{x} \in \mathbb{C}^{n}} & \|\nabla \bar{x}\|_{1} \\
\text { s.t. } & \left\|z_{I Q}-S(h \otimes \bar{x})\right\|_{2}^{2} \leq \alpha
\end{array}
$$

or in its equivalent unconstrained form:

$$
\min _{\bar{x} \in \mathbb{C}^{n}}\||\nabla \bar{x}|\|_{1}+\tau\left\|z_{I Q}-S(H \otimes \bar{x})\right\|_{2}^{2},
$$

The temporal notation is removed for simplicity. $S$ is a simple structure matrix depicting a $s$ factor downsampling in vertical and horizontal directions, $\nabla=\left(\nabla_{v}, \nabla_{h}\right)^{\mathrm{T}}: \mathbb{C}^{n} \rightarrow \mathbb{C}^{n} \times \mathbb{C}^{n}$ is a discrete version of the vertical and horizontal gradients, $\tau$ and $\alpha$ are positive real numbers measuring the trade-off between the fit to $z_{I Q}$ and the amount of TV regularization. Note that since we choose circular boundary conditions, $H$, $\nabla_{v}$ and $\nabla_{h}$ are block-circulant matrices and can hence be diagonalized by Fourier Transform. Note that $\nabla x \in \mathbb{C}^{n} \times \mathbb{C}^{n}$ means that $\nabla x$ is a matrix with 2 rows and $n$ columns. $\|\cdot\|_{2}$ refers to the standard definition of an $\mathcal{L}^{2}$-norm, while \|\|$\cdot\|\|_{1}$ refers to another $\mathcal{L}^{1}$-norm.

The super-resolution task is hence formulated as a constrained optimization problem expressed in (6).

\subsection{General Alternating Direction Method of Multipliers Framework}

Many numerical algorithms are able to solve equation (6), and the conjugate gradient methods would be the most simple and popular amongst them. Unlike the ADMM framework, such schemes are known to be slow to converge, especially when the functional is not quadratic, non-smooth or when its gradient is not Lipchitz ${ }^{1}$ differentiable [8].

Let us consider the following real case general optimization problem:

$$
\begin{array}{cl}
\min & f_{1}(x)+f_{2}(y) \\
\text { s.t. } & x \in \mathcal{X}, y \in \mathcal{Y}, A x+B y=c
\end{array}
$$

where $A \in \mathcal{M}_{l, n}(\mathbb{R})$ and $B \in \mathcal{M}_{l, m}(\mathbb{R})$ are $l \times n$ and $l \times m$ given matrices, respectively; $c \in \mathbb{R}^{l}$ is a given vector, $\mathcal{X} \subseteq$ $\mathbb{R}^{n}$ and $\mathcal{Y} \subseteq \mathbb{R}^{m}$ are given convex sets, $f_{1}: X \rightarrow \mathbb{R}^{n}$ and $f_{2}: Y \rightarrow \mathbb{R}^{m}$ are closed convex functions.

The Augmented Lagrangian (AL) function of (8) writes

$$
\begin{aligned}
\mathcal{L}(x, y, \lambda) & =f_{1}(x)+f_{2}(y)+ \\
& \langle\lambda, A x+B y-c\rangle+\frac{\beta}{2}\|A x+B y-c\|_{2}^{2},
\end{aligned}
$$

where $\lambda \in \mathbb{R}^{l}$ is the Lagrangian multiplier attached to the linear constraints, $\beta \in \mathbb{R}^{+*}$ is the penalty parameter for the violation of theses constraints and $\langle\cdot, \cdot\rangle$ denotes the usual inner product.

Given an initial $\lambda_{0}$, the AL method approaches the solution of the original problem (8) by iteratively solving the following auxiliary problem :

$$
\left\{\begin{aligned}
\left(x_{k+1}, y_{k+1}\right) & =\underset{x \in \mathcal{X}, y \in \mathcal{Y}}{\operatorname{argmin}} \mathcal{L}\left(x, y, \lambda_{k}\right) \\
\lambda_{k+1} & =\lambda_{k}+\beta\left(A x_{k+1}+B y_{k+1}-c\right)
\end{aligned}\right.
$$

However, despite its popularity for solving generic optimization problems with such constraints, it would not be judicious to implement directly the original AL method (10) to solve our specific problem (8). The separable structure emerging in both the objective function and the constraint would completely be omitted, thus requiring the simultaneous minimization of $x_{k+1}$ and $\mathrm{y}_{k+1}$ in (10). Hopefully, thanks to the ADMM framework, we rather iteratively solve the following three stage problem:

$$
\left\{\begin{array}{lll}
x_{k+1} & =\underset{x \in \mathcal{X}}{\operatorname{argmin}} & \mathcal{L}\left(x, y_{k}, \lambda_{k}\right) \\
y_{k+1} & =\underset{y \in \mathcal{Y}}{\operatorname{argmin}} \mathcal{L}\left(x_{k+1}, y, \lambda_{k}\right) \\
\lambda_{k+1} & =\lambda_{k}+\beta\left(A x_{k+1}+B y_{k+1}-c\right)
\end{array}\right.
$$

ADM hence inherits the algorithmic framework of the AL method but with the improvement of minimizing $x_{k+1}$

\footnotetext{
${ }^{1}$ A function $f: X \mapsto Y$ is $\mathrm{K}$-Lipschitz continuous if there exist a real constant $K \geq 0$ such that $\forall x_{1}, x_{2} \in X,\left|f\left(x_{1}\right)-f\left(x_{2}\right)\right| \leq K\left|x_{1}-x_{2}\right|$.
} 
and $\mathrm{y}_{k+1}$ serially via solving two lower-dimensional subproblems. Our problem can eventually be reformulated as follows:

$$
\begin{array}{cl}
\min & \||\omega|\|_{1} \\
\text { s.t. } & \bar{x} \in \mathbb{C}^{n}, \omega \in \mathbb{C}^{n} \times \mathbb{C}^{n}, \omega=\nabla \bar{x}, z \in \mathcal{Z}
\end{array}
$$

where $\mathcal{Z}=\left\{z \in \mathbb{C}^{n}, z=h \otimes \bar{x},\|e-S(z)\|_{2}^{2} \leq \alpha\right\}$.

This problem fits the framework (8) by choosing $y=$ $(\omega, z)^{\mathrm{T}}, f_{1}(x)=0, f_{2}(y)=\|\| \omega \|_{1}+\chi_{\mathcal{Z}}(z), A=(\nabla, H)^{\mathrm{T}}$, $B=\left(-I_{\mathbb{C}^{n} \times \mathbb{C}^{n}},-I_{\mathbb{C}^{n}}\right)^{\mathrm{T}}$ and $c=0 . I_{\mathbb{C}^{n}}$ denotes the $\mathbb{C}^{n}$ identity matrix and $\chi_{\mathcal{Z}}$ the indicator function of $\mathcal{Z}$.

\subsection{Detailed Implementation}

According to the previous framework, the AL (9) can be rewritten as:

$$
\mathcal{L}(x, y, \lambda)=f_{2}(y)+\langle\lambda, A x+B y\rangle+\frac{\beta}{2}\|A x+B y\|_{2}^{2},
$$

Note that $l=3 n$ and $\lambda=\left(\lambda_{1}, \lambda_{2}, \lambda_{3}\right)^{\mathrm{T}} \in \mathbb{C}^{n} \times \mathbb{C}^{n} \times \mathbb{C}^{n}$, as well as $y$. The three step ADMM algorithm comes after some algebraic development:

- Step 1:

$$
\begin{aligned}
x_{k+1} & =\underset{x \in \mathbb{C}^{n}}{\operatorname{argmin}} \mathcal{L}\left(x, y_{k}, \lambda_{k}\right) \\
& =\underset{x \in \mathbb{C}^{n}}{\operatorname{argmin}}\left\langle\lambda_{k}, A x+B y_{k}\right\rangle+\frac{\beta}{2}\left\|A x+B y_{k}\right\|_{2}^{2}
\end{aligned}
$$

$x_{k+1}$ is the solution of a linear system and can be easily retrieved in the spectral domain, where operations are done pointwise using Fast Fourier Transform (FFT) and Inverse Fast Fourier Transform (IFFT), written $\mathcal{F}\{\cdot\}$ and $\mathcal{F}^{-1}\{\cdot\}$ :

$$
x_{k+1}=\mathcal{F}^{-1}\left\{\frac{\mathcal{F}\left\{\beta\left[\nabla^{T}\left(\left(y_{1, k}, y_{2, k}\right)-\frac{\left(\lambda_{1, k}, \lambda_{2, k}\right)}{\beta}\right)+H^{T}\left(y_{3, k}-\frac{\lambda_{3, k}}{\beta}\right)\right]\right\}}{\mathcal{F}\left\{\nabla^{T} \nabla+H^{T} H\right\}}\right\}
$$

- Step 2:

$$
\begin{aligned}
y_{k+1} & =\underset{y \in \mathbb{C}^{n} \times \mathbb{C}^{n} \times \mathbb{C}^{n}}{\operatorname{argmin}} \mathcal{L}\left(x_{k+1}, y, \lambda_{k}\right) \\
& =\underset{y \in \mathbb{C}^{n} \times \mathbb{C}^{n} \times \mathbb{C}^{n}}{\operatorname{argmin}} f_{2}(y)+\frac{\beta}{2}\left\|y-\left(A x_{k+1}+\frac{\lambda_{k}}{\beta}\right)\right\|_{2}^{2}
\end{aligned}
$$

which can be split into $y_{k+1}=\left(\omega_{k+1}, z_{k+1}\right)^{\mathrm{T}}$ and solved as:

$$
\left\{\begin{array}{l}
\omega_{k+1}=\operatorname{soft}_{\frac{1}{\beta}}\left(\nabla x_{k+1}+\frac{\left(\lambda_{1, k}, \lambda_{2, k}\right)}{\beta}\right) \\
S\left(z_{k+1}\right)=\frac{S\left(H x_{k+1}+\frac{\lambda_{3, k}}{\beta}\right)+2 \gamma x_{0}}{1+2 \gamma}
\end{array}\right.
$$

where $\gamma=\frac{1}{2}+\frac{1}{2} \sqrt{\frac{C}{\alpha}}$, with $C$ a positive constant solution of a quadratic equation not explicitly reproduced here for simplicity and $\alpha$ the trade-off constant defined in (6). soft $(\cdot)$ is the (complex) soft-thresholding operator defined for complex argument by

$$
\operatorname{soft}_{\delta}(\omega)=\omega \frac{\max (|\omega|-\delta, 0)}{\max (|\omega|-\delta, 0)+\delta} .
$$

- Step 3:

$$
\lambda_{k+1}=\lambda_{k}+\beta\left(A x_{k+1}+B y_{k+1}\right) .
$$

\section{EXPERIMENTS, RESULTS AND DISCUSSION}

\subsection{Point Spread Function}

Estimation of USI PSF is a crucial step in the restoration process and inaccuracies during this stage may cause a severe decrease in the algorithm general performances. Besides, the PSF $g(t)$ can be effectively estimated from the RF signal based on the minimum phase assumption [10]. For the sake of simplicity, we will here consider a space-invariant PSF.

\subsection{Synthetic Images}

Synthetic images were generated using FIELD II [11] in order to determine both the axial and lateral resolutions. Note that equation (6) becomes much simpler when the input signal $\bar{x}$ is an impulsion.

The PSF was first considered space-invariant and approximated by the inpulse response of a point source within the focal zone. Fig. 1 shows (a) the original image and (b) the degraded image, blurred using the previously described kernel, downsampled with $s=2$ and with a slight $20 \mathrm{~dB}$ SNR noise added. (c) and (d) are the results of the $\mathcal{L}^{1}$ criterion [9] based algorithm and the proposed TV criterion based ADMM, respectively. In this simple case, it is better to use the sparsity hypothesis

\subsection{In Vivo Ultrasound Images}

Ultrasound image of a mouse kidney acquired with a $25 \mathrm{MHz}$ transducer was processed. As shown by the results presented in Fig. 2, the restoration effect yields a significant qualitative improvement in both the signal-to-noise ratio and the contrast of the biological sample. (a) and (b) are two input images of the mouse kidney, (c) and (d) are the corresponding results performed with the Chambolle algorithm, (e) and (f) are the corresponding TV-ADMM results performed with our method.

From these figures it can be noticed that the sparsity hypothesis can not stand for such US images. To our knowledge, there is not any simple SR method for USI: this is why the comparison is performed with the USI deconvolution framework in [12] which is reported to be one of the most popular technique.

\section{CONCLUSION}

This paper has proposed an ADMM based technique for the restoration of US images and the resolution improvement is observable on synthetic and in vivo US images. Further works 
will deal with improved space-variant PSF estimation and accounting for the speckle statistic of US images together with quantitative evaluation and deeper comparisons.

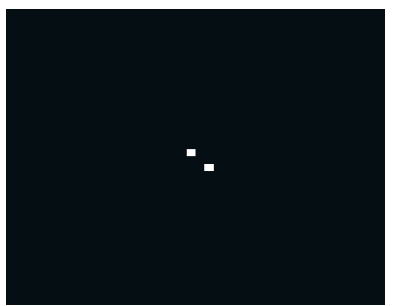

(a) Original image

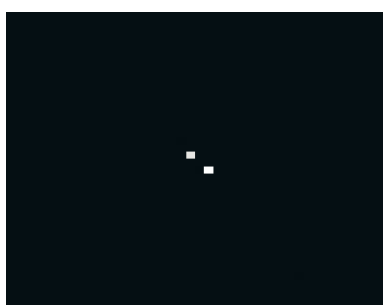

(c) $\mathcal{L}^{1}$ result

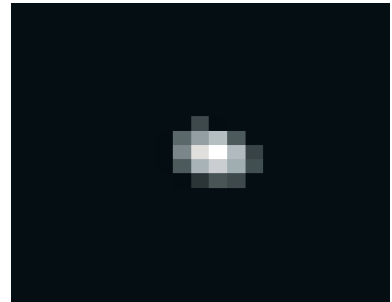

(b) Contaminated image

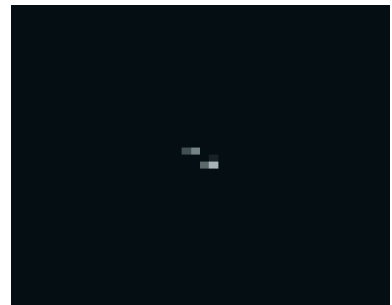

(d) TV-ADMM result
Fig. 1. Resolution of two close point sources with parameters $\alpha=0.1, \beta=0.8$. $s$ was set to 2 . The convolution kernel is simulated using FIELD II and a $3 \mathrm{MHz}$ center frequency linear transducer. (a) and (b) are the original and contaminated images respectively, (c) and (d) are the $\mathcal{L}^{1}$-ADMM and TV-ADMM result image

\section{REFERENCES}

[1] T. L. Szabo, Diagnostic ultrasound imaging: inside out, Biomedical Engineering Series. Elsevier Academic Press, 2004.

[2] J. L. Prince and J. M. Links, Medical imaging signals and systems, Pearson Prentice Hall, 2006.

[3] G. T. Clement, J. Huttunen, and K. Hynynen, "Superresolution ultrasound imaging using back-projected reconstruction," $J$. Acoust. Soc. Am., vol. 118, no. 6, pp. 3953 - 3960, Sept. 2005.

[4] M. A. Ellis, F. V., and W. F. Walker, "Super-resolution image reconstruction using diffuse source models," Ultrasound in Med. \& Biol., vol. 36, no. 6, pp. 967 - 977, 2010.

[5] O. Michailovich and A. Tannenbaum, "Blind deconvolution of medical ultrasound images: A parametric inverse filtering approach," IEEE Trans. Image Processing, vol. 16, no. 12, pp. 3005 - 3019, Dec. 2007.

[6] T. Taxt, "Restoration of medical ultrasound images using twodimensional homomorphic deconvolution," IEEE Trans. Ultrason. Ferroelectr. Freq. Control, vol. 42, no. 4, pp. 543 - 554, July 1995.

[7] L. I. Rudin, S. Osher, and E. Fatemi, "Nonlinear total variation based noise removal algorithms," Physica D: Nonlinear Phenomena, vol. 60, pp. $259-268,1992$.

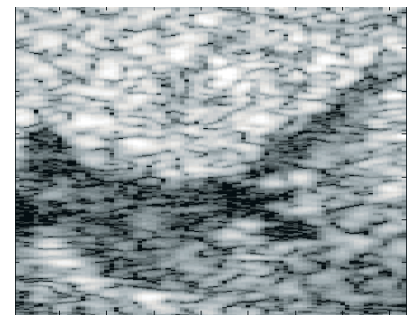

(a) Input image 1

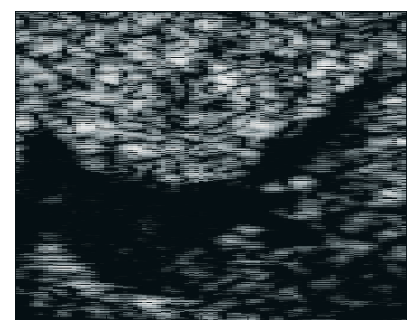

(c) Chambolle result 1

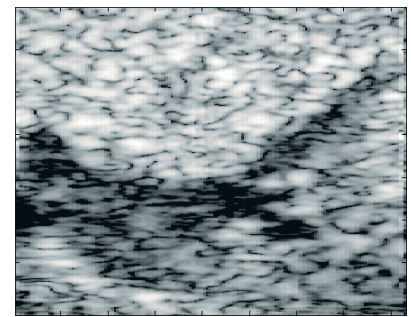

(c) TV-ADMM result 1

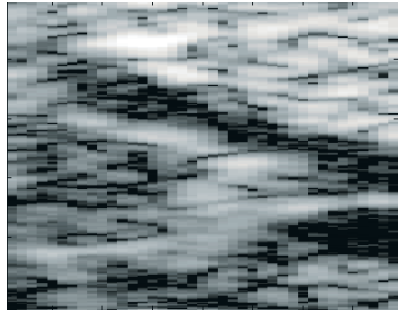

(b) Input image 2

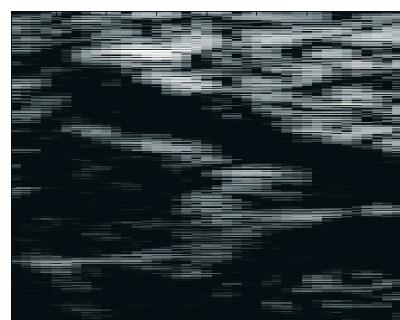

(d) Chambolle result 2

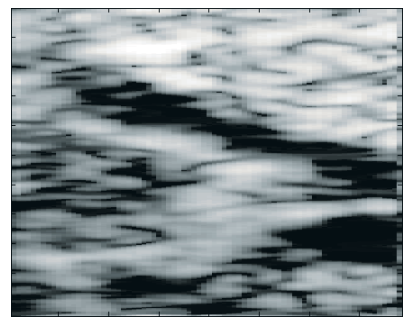

(d) TV-ADMM result 2
Fig. 2. ADMM restoration of a mouse kidney. Transducer central frequency is $25 \mathrm{MHz}$. Figure shows (top row) the input images, (middle row) the Chambolle algorithm result images and (bottom row) the TV-ADMM result images.

[8] M. Ng, P. Weiss, and X.-M. Yuang, "Solving constrained totalvariation image restoration and reconstruction problems via alternating direction methods," SIAM journal on Scientific Computing, vol. 32, 2010.

[9] C. Yu, C. Zhang, and L. Xie, "An envelope signal based deconvolution algorithm for ultrasound imaging," Signal Processing, vol. 92, no. 3, pp. $793-800,2012$.

[10] J. A. Jensen and S. Leeman, "Nonparametric estimation of ultrasound pulses," IEEE Trans. Biomedical Engineering, vol. 41, no. 10, pp. 929 - 936, Oct. 1994.

[11] J. A. Jensen, "A model for the propagation and scattering of ultrasound in tissue," J. Acoust. Soc. Am., vol. 89, no. 1, pp. 182 - 191, Jan. 1991.

[12] Antonin Chambolle, "An algorithm for total variation minimization and applications," J. Math. Imag. Vision, vol. 20, pp. $89-97,2004$ 